\title{
Nonexistence results of Caputo-type fractional problem
}

\author{
Mohammed D. Kassim ${ }^{1}$, Saeed M. Ali ${ }^{1}$ (D), Mohammed S. Abdo ${ }^{2}$ (D) and Fahd Jarad ${ }^{3,4^{*}}$ (ID
}

\author{
${ }^{*}$ Correspondence: \\ fahd@cankaya.edu.tr \\ ${ }^{3}$ Department of Mathematics, \\ Cankaya University, 06790 \\ Etimesgut, Ankara, Turkey \\ ${ }^{4}$ Medical University Hospital, China \\ Medical University, Taichung, \\ Taiwan \\ Full list of author information is \\ available at the end of the article

\begin{abstract}
In this paper, we deal with Caputo-type fractional differential inequality where there is a low-order fractional derivative with the term polynomial source. We investigate the nonexistence of nontrivial global solutions in a suitable space via the test function technique and some properties of fractional integrals. Finally, we demonstrate three examples to illustrate our results. The presented results are more general than those in the literature, which can be obtained as particular cases.
\end{abstract}

MSC: Primary 26A33; secondary 34A07; 93A30; 35R11

Keywords: Nonexistence; Caputo fractional derivative; Global solution; Test function method

\section{Introduction}

Fractional-order differential equations have more benefits in contrast with integer-order differential equations. These equations are adaptable and exact in portraying the changing law of things. Hence fractional-order differential equations are broadly utilized in real life [1-6]. Nonetheless, some physical meanings of the fractional differential equations (FDEs) are yet to be generally perceived because of the intricacy of its initial values; consequently, the improvement of the theory of FDEs is as yet in its early stages. However, these equations have become a significant point among numerous researchers in light of their wide practical applications and theoretical importance.

Although fractional calculus was proposed 300 years ago, the scientists and researchers are still developing and building up this field significantly, as it is closely related to many other disciplines. Because of the significance of fractional calculus in applications, in the previous few decades, there has been a developing interest in the investigation of FDEs. Specifically, from the theoretical perspective, the existence of solutions for various classes of FDEs was discussed in numerous contributions (see, e.g., [7-19]). As applications, there are many recent models and numerical results regarding several classes of FDEs involving various types of fractional derivatives (FDs) [20-26]. Regarding the problem of the nonexistence of solutions for FDEs, we refer to [27-36]. In this regard, we consider the following

(c) The Author(s) 2021. This article is licensed under a Creative Commons Attribution 4.0 International License, which permits use sharing, adaptation, distribution and reproduction in any medium or format, as long as you give appropriate credit to the original author(s) and the source, provide a link to the Creative Commons licence, and indicate if changes were made. The images or other third party material in this article are included in the article's Creative Commons licence, unless indicated otherwise in a credit line to the material. If material is not included in the article's Creative Commons licence and your intended use is not permitted by statutory regulation or exceeds the permitted use, you will need to obtain permission directly from the copyright holder. To view a copy of this licence, visit http://creativecommons.org/licenses/by/4.0/. 
problem:

$$
\left\{\begin{array}{l}
{ }^{C} D_{0}^{\lambda} \varkappa(t)+{ }^{C} D_{0}^{\gamma} \varkappa(t)=\mathfrak{F}(t, \varkappa(t)), \quad t>0, \\
\varkappa^{(i)}(0)=b_{i}, \quad i=0,1, \ldots, n-1, n=-[-\lambda],
\end{array}\right.
$$

where ${ }^{C} D_{a}^{\sigma}$ is the Caputo derivative of order $\sigma(>0) \in\{\lambda, \gamma\}, n<\lambda, \gamma<n+1(n \in \mathbb{N})$.

We will highlight the nonexistence result of nontrivial global solutions for (1) along with the following condition:

$$
\mathfrak{F}(t, \varkappa(t)) \geq t^{\eta}\left|{ }^{C} D_{0}^{\vartheta} \varkappa(t)\right|^{m}, \quad \lambda=\kappa+1, \quad \gamma=v+1,
$$

for some $m>1, \eta \in \mathbb{R}$, and $n-1<\lambda, \gamma, \vartheta<n(n \in \mathbb{N})$. That is, we regard the following problem:

$$
\left\{\begin{array}{l}
{ }^{C} D_{0}^{\kappa+1} \varkappa(t)+{ }^{C} D_{0}^{v+1} \varkappa(t) \geq t^{\eta}\left|{ }^{C} D_{0}^{\vartheta} \varkappa(t)\right|^{m}, \quad t>0, m>1, \\
\varkappa^{(i)}(0)=b_{i}, \quad i=0,1, \ldots, n, n=-[-\kappa],
\end{array}\right.
$$

where $\kappa, v, \vartheta \in(n-1, n), n \in \mathbb{N}$, and we show that there are no solutions for specific values of $\eta$ and $m$. Specifically, we discover the range of values of $m$ for which solutions do not exist globally. Obviously, sufficient conditions for nonexistence give necessary conditions for the existence of solutions.

Remark 1 The existence and uniqueness of solutions for problem (1) was discussed in [4].

Remark 2 In the case $\kappa=v=\vartheta=0$ in (2), we obtain the problem

$$
\varkappa^{\prime}(t)=t^{\eta} \varkappa^{m}(t), \quad \varkappa(0)=b,
$$

which has a solution

$$
\varkappa(t)=\left[\frac{1-m}{1+\eta} t^{1+\eta}+b^{1-m}\right]^{1 /(1-m)} \quad \text { for } m>1
$$

Notice that the solution blows up in finite time for $m>1$.

Remark 3 In case $\lambda=1, \gamma=0$, and $\mathfrak{F}(t, \varkappa(t))=\varkappa^{m}(t), m>1$ in (1), we obtain the Bernoulli differential problem

$$
\varkappa^{\prime}(t)+\varkappa(t)=\varkappa^{m}(t), \quad \varkappa(0)=b,
$$

which has a solution

$$
\varkappa(t)=\left[1+\left(b^{1-m}-1\right) \exp (m-1) t\right]^{1 /(1-m)} .
$$

Obviously, $\varkappa(t)$ blows up in the finite time $c=\frac{1}{1-m} \ln \left(1-b^{1-m}\right)$ for $m, b>1$. 
Remark 4 In the case $\lambda=\gamma=\kappa$ and $\mathfrak{F}(t, \varkappa(t)) \geq t^{\vartheta}|\varkappa(t)|^{m}$ in (1), we obtain

$$
\left\{\begin{array}{l}
2 D_{0}^{\kappa} \varkappa(t) \geq t^{\vartheta}|\varkappa(t)|^{m}, \quad t>0 \\
\left.I_{0}^{1-\kappa} \varkappa(t)\right|_{t=0}=b
\end{array}\right.
$$

Problem (3) was taken into consideration by Laskri and Tatar [29]. It turns out that if $\vartheta>-\kappa$ and $1<m \leq \frac{\vartheta+1}{1-\kappa}$, then problem (3) admits no global nontrivial solutions if $b \geq 0$.

Kassim et al. [35] studied the problem

$$
\left\{\begin{array}{l}
{ }^{C} D_{0}^{\kappa} \varkappa(t)+{ }^{C} D_{0}^{v} \varkappa(t) \geq t^{\vartheta}|\varkappa(t)|^{m}, \quad t>0, \\
\varkappa^{(k)}(0)=b_{k}, \quad k=0,1, \ldots, n-1,
\end{array}\right.
$$

where $m>1, n \geq 1$ is an integer, $n-1<v \leq \kappa<n$, and $b_{k} \geq 0$. It turns out that if $m(1-$ $v)-1<\vartheta<m-1$, then problem (4) admits no nontrivial global solutions.

In case $0<\kappa, v, \vartheta<1$ and $\eta=0$ in (2), we obtain

$$
\left\{\begin{array}{l}
{ }^{C} D_{0}^{\kappa+1} \varkappa(t)+{ }^{C} D_{0}^{v+1} \varkappa(t) \geq\left|{ }^{C} D_{0}^{\vartheta} \varkappa(t)\right|^{m}, \quad t>0, \\
\varkappa(0)=b_{0}, \quad \varkappa^{\prime}(0)=b_{1} .
\end{array}\right.
$$

Not long ago, Jleli and Samet [37] studied (5). They proved that the problem admits no global solutions if $b_{1}>0$.

In this work, we investigate the case of a lower order FD in the inequality (or equation). It is clear that for hyperbolic equations, for example, the wave equation with an interval fractional damping represented by the first derivative (i.e., $\kappa=1, v=0$ ), this damping process has a squandering effect. It will contend with the polynomial source and may take care of this blowing-up term under certain conditions. Besides, in the telegraphing problem [38], the solutions approach the solution of the same problem without the $n$th derivative as $t \rightarrow \infty$ (i.e., the parabolic equation). This result has been summed up and generalized to the FD case in [38] and [30]. For our concern with a problem (2), we might want to see how effective $D_{0}^{v} \varkappa$ will be on the blowup phenomenon, specifically, how the range of values $m$ guaranteeing to blowup in finite time would be influenced. We arrived at the conclusion that here it is the lower-order derivative (i.e., $v$ ), which determines the range of blowup much the same as the parabolic portion and hyperbolic problem.

In Sect. 2, we give some notations, definitions, and lemmas required later in our analysis. Sections 3 and 4 are committed to the test function and the nonexistence result. In Sect. 5, we provide some examples to justify the preceding results. In the final section, we close our work with concluding remarks.

\section{Preliminaries}

In this section, we recall some primary facts utilized in our outcomes. We refer the reader to [4-6] for additional insights about FDs.

Definition 1 We denote by $A C[0, \infty)$ the space of absolutely continuous functions on $[0, \infty)$ and by $A C^{n}[0, \infty)$ the space of functions $\varpi$ that have continuous derivatives up to order $n-1$ on $[0, \infty)$ such that $\varpi^{(n-1)} \in A C[0, \infty)$, where $\varpi^{(n-1)}$ denotes the derivative of order $n-1$ of $\varpi$. 
Definition 2 We denote by $L_{p}(a, b), p \geq 1$, the spaces of Lebesgue-integrable functions on $(a, b)$.

Definition 3 Let $a<t<b$ and $\kappa>0$, and let $\varpi \in L_{1}(a, b)$. Then the left- and right-sided Riemann-Liouville fractional integrals of order $\kappa$ of $\varpi$ are given by

$$
\left(I_{a}^{\kappa} \varpi\right)(t):=\frac{1}{\Gamma(\kappa)} \int_{a}^{t} \frac{\varpi(r)}{(t-r)^{1-\kappa}} d r
$$

and

$$
\left(I_{b-}^{\kappa} \varpi\right)(t):=\frac{1}{\Gamma(\kappa)} \int_{t}^{b} \frac{\varpi(r)}{(r-t)^{1-\kappa}} d r
$$

respectively, where $\Gamma$ is the gamma function. Note that if $\kappa=0$, then $I_{a}^{0} \varpi=I_{b-}^{0} \varpi=\varpi$.

Definition 4 Let $\varpi \in A C^{n}[0, \infty)$. The expression

$$
{ }^{C} D_{a}^{\kappa} \varpi(t)=I_{a}^{n-\kappa} \varpi^{(n)}(t)=\frac{1}{\Gamma(n-\kappa)} \int_{a}^{t} \frac{\varpi^{(n)}(s)}{(t-s)^{\kappa+1-n}} d s
$$

is called left-sided Caputo FD of order $\kappa$ of $\varpi$.

Lemma 1 ([6]) Let $\kappa>0, p \geq 1, q \geq 1$, and $\frac{1}{p}+\frac{1}{q} \leq 1+\kappa(p \neq 1$ and $q \neq 1$ in the case where $\left.\frac{1}{p}+\frac{1}{q}=1+\kappa\right)$. If $\Theta_{1} \in L_{p}(a, b)$ and $\Theta_{2} \in L_{q}(a, b)$, then

$$
\int_{a}^{b} \Theta_{1}(t)\left(I_{a}^{\kappa} \Theta_{2}\right)(t) d t=\int_{a}^{b} \Theta_{2}(t)\left(I_{b-}^{\kappa} \Theta_{1}\right)(t) d t
$$

Lemma 2 If $\kappa \geq 0$ and $v>0$, then

$$
\begin{aligned}
& I_{T}^{\kappa}(T-t)^{\kappa}=\frac{\Gamma(v+1)}{\Gamma(v+\kappa+1)}(T-t)^{v+\kappa}, \\
& D_{T}^{\kappa}(T-t)^{v}=\frac{\Gamma(v+1)}{\Gamma(v-\kappa+1)}(T-t)^{v-\kappa} .
\end{aligned}
$$

Lemma 3 Let $\kappa>0$ and $v>0$. If $\varpi \in L_{p}(a, b)$, then

$$
\begin{array}{ll}
I_{a}^{v} I_{a}^{\kappa} \varpi(t)=I_{a}^{v+\kappa} \varpi(t), & t>a, \\
I_{b}^{v} I_{b}^{\kappa} \varpi(t)=I_{b}^{v+\kappa} \varpi(t), & t<b .
\end{array}
$$

Lemma 4 Let $\kappa \in(n-1, n), n \in \mathbb{N}$, and $\varpi \in L_{p}(0, b)$. Then the IVP

$$
\begin{aligned}
& { }^{C} D_{0}^{\kappa} \varkappa(t)=\varpi(t), \quad t>0, \\
& \varkappa^{(i)}(0)=c_{i}, \quad i=0,1, \ldots, n-1,
\end{aligned}
$$

has the solution

$$
\varkappa(t)=c_{0}+c_{1} t+\cdots+\frac{c_{n-1}}{(n-1) !} t^{n-1}+\frac{1}{\Gamma(\kappa)} \int_{0}^{t}(t-r)^{\kappa-1} \varpi(r) d r .
$$




\section{The test function}

We use the test function

$$
\Theta(t)=\left\{\begin{array}{l}
T^{-\varsigma}(T-t)^{\varsigma}, \quad 0 \leq t \leq T, \varsigma>0, \\
0, \quad t>T .
\end{array}\right.
$$

This test function has the following properties.

Lemma 5 Let $\eta \geq 0$ and $\Theta$ be as in (10). Then

$$
\begin{aligned}
& I_{T}^{\eta} \Theta(t)=\frac{\Gamma(\varsigma+1)}{\Gamma(\eta+\varsigma+1)} T^{-\varsigma}(T-t)^{\varsigma+\eta}, \\
& I_{T}^{\eta} \Theta(0)=\frac{\Gamma(\varsigma+1)}{\Gamma(\eta+\varsigma+1)} T^{\eta} \text { and } I_{T}^{\eta} \Theta(T)=0 \\
& \frac{d^{2}}{d t^{2}} I_{T}^{\eta} \Theta(t)=\frac{\Gamma(\varsigma+1)}{\Gamma(\eta+\varsigma-1)} T^{-\varsigma}(T-t)^{\varsigma+\eta-2} .
\end{aligned}
$$

Proof It follows from Lemma 2.

Lemma 6 Let $\Theta$ be as in (10) with $\varsigma+p(v+\kappa-2)+1>0, \vartheta(1-p)+1>0$, $v, \kappa \geq 0$, and $p>1$. Then

$$
\int_{0}^{T} t^{\vartheta(1-p)} \Theta^{1-p}(t)\left(I_{T}^{v} \frac{d^{2}}{d t^{2}} I_{T}^{\kappa} \Theta(t)\right)^{p} d t=K_{v, \kappa}^{\vartheta, p, \varsigma} T^{\vartheta(1-p)+p(v+\kappa-2)+1},
$$

where

$$
K_{v, \kappa}^{\vartheta, p, \varsigma}=\left[\frac{\Gamma(\varsigma+1)}{\Gamma(v+\kappa+\varsigma-1)}\right]^{p} \frac{\Gamma(\vartheta(1-p)+1) \Gamma(\varsigma+p(v+\kappa-2)+1)}{\Gamma(\vartheta(1-p)+\varsigma+p(v+\kappa-2)+2)} .
$$

Proof By Lemma 5 we have

$$
\begin{aligned}
I_{T}^{v} \frac{d^{2}}{d t^{2}} I_{T}^{\kappa} \Theta(t) & =\frac{\Gamma(\varsigma+1)}{\Gamma(\kappa+\varsigma-1)} T^{-\varsigma} I_{T}^{v}(T-t)^{\varsigma+\kappa-2} \\
& =\frac{\Gamma(\varsigma+1)}{\Gamma(v+\kappa+\varsigma-1)} T^{-\varsigma}(T-t)^{v+\varsigma+\kappa-2} .
\end{aligned}
$$

Then

$$
\begin{aligned}
\int_{0}^{T} t^{\vartheta(1-p)} \Theta^{1-p}(t)\left(I_{T}^{v} \frac{d^{2}}{d t^{2}} I_{T}^{\kappa} \Theta(t)\right)^{p} d t \\
\quad=\left[\frac{\Gamma(\varsigma+1)}{\Gamma(v+\kappa+\varsigma-1)}\right]^{p} T^{-p \varsigma} \int_{0}^{T} t^{\vartheta(1-p)}\left[T^{-\varsigma}(T-t)^{\zeta}\right]^{1-p}(T-t)^{p(v+\varsigma+\kappa-2)} d t \\
\quad=\left[\frac{\Gamma(\varsigma+1)}{\Gamma(v+\kappa+\varsigma-1)}\right]^{p} T^{-\varsigma} \int_{0}^{T} t^{\vartheta(1-p)}(T-t)^{\zeta+p(v+\kappa-2)} d t .
\end{aligned}
$$

Let $t=s T$. Then

$$
\int_{0}^{T} t^{\vartheta(1-p)} \Theta^{1-p}(t)\left(I_{T}^{v} \frac{d^{2}}{d t^{2}} I_{T}^{\kappa} \Theta(t)\right)^{p} d t
$$




$$
\begin{aligned}
& =\left[\frac{\Gamma(\varsigma+1)}{\Gamma(v+\kappa+\varsigma-1)}\right]^{p} T^{\vartheta(1-p)+p(v+\kappa-2)+1} \int_{0}^{1} s^{\vartheta(1-p)}(1-s)^{\varsigma+p(v+\kappa-2)} d s \\
& =\left[\frac{\Gamma(\varsigma+1)}{\Gamma(v+\kappa+\varsigma-1)}\right]^{p} \frac{\Gamma(\vartheta(1-p)+1) \Gamma(\varsigma+p(v+\kappa-2)+1)}{\Gamma(\vartheta(1-p)+\varsigma+p(v+\kappa-2)+2)} T^{\vartheta(1-p)+p(v+\kappa-2)+1} .
\end{aligned}
$$

Remark 5 For the rest of the paper, we will utilize the following equivalency. If $m, m^{\prime}>1$, and $\frac{1}{m}+\frac{1}{m^{\prime}}=1$, then:

1. $m^{\prime}=\frac{m}{m-1}$.

2. $\frac{m^{\prime}}{m}=m^{\prime}-1$.

3. $m(\kappa-1)+1>0 \Longleftrightarrow m^{\prime} \kappa>1$ for $\kappa>0$.

\section{Nonexistence result}

In this part, we discuss the problem

$$
\left\{\begin{array}{l}
{ }^{C} D_{0}^{\kappa+1} \varkappa(t)+{ }^{C} D_{0}^{v+1} \varkappa(t) \geq t^{\eta}\left|{ }^{C} D_{0}^{\vartheta} \varkappa(t)\right|^{m}, \quad t>0, m>1,0<v<\kappa, \\
\varkappa^{(i)}(0)=b_{i}, \quad i=0,1, \ldots, n, n=-[-\kappa] .
\end{array}\right.
$$

Theorem 1 Let $m(\vartheta-n)+n-v-1<\eta<m-1$ and $m>1$. If $b_{n}>0$, then problem (11) admits no global nontrivial solutions in $A C^{n+1}[0, \infty)$.

Proof Let $\varkappa \in A C^{n+1}[0, \infty)$ be a global solution to (11). Let $\Theta$ be as in (10) with $\varsigma>\frac{m}{m-1}(\kappa+$ $1-\vartheta)-1$. Multiplying both sides of $(11)$ by $\Theta(t)$ and integrating over $[0, T]$, we get

$$
I=\int_{0}^{T} \Theta(t) t^{\eta}\left|{ }^{C} D_{0}^{\vartheta} \varkappa(t)\right|^{m} d t \leq I_{1}+I_{2}
$$

where

$$
I_{1}=\int_{0}^{T} \Theta(t)^{C} D_{0}^{\kappa+1} \varkappa(t) d t
$$

and

$$
I_{2}=\int_{0}^{T} \Theta(t)^{C} D_{0}^{v+1} \varkappa(t) d t
$$

From the definition of ${ }^{C} D_{0}^{\vartheta} \varkappa(t)$ and Lemma 1 we have

$$
I_{1}=\int_{0}^{T} \Theta(t) I_{0}^{n-\kappa} \varkappa^{(n+1)}(t) d t=\int_{0}^{T} \varkappa^{(n+1)}(t) I_{T}^{n-\kappa} \Theta(t) d t
$$

Integrating by parts and using Lemma 5 , we get

$$
\begin{aligned}
I_{1} & =\left.\varkappa^{(n)}(t) I_{T}^{n-\kappa} \Theta(t)\right|_{t=0} ^{T}-\int_{0}^{T} \varkappa^{(n)}(t) \frac{d}{d t} I_{T}^{n-\kappa} \Theta(t) d t \\
& =-b_{n} I_{T}^{n-\kappa} \Theta(0)-\int_{0}^{T} \varkappa^{(n)}(t) \frac{d}{d t} I_{T}^{n-\kappa} \Theta(t) d t .
\end{aligned}
$$


On the other hand, by Lemma 3 we obtain

$$
\begin{aligned}
\varkappa^{(n)}(t) & =\frac{d}{d t} \int_{0}^{t} \varkappa^{(n)}(s) d s=\frac{d}{d t}\left(I_{0}^{1} \varkappa^{(n)}(t)\right) \\
& =\frac{d}{d t}\left(I_{0}^{\vartheta+1-n} I_{0}^{n-\vartheta} \varkappa^{(n)}(t)\right)=\frac{d}{d t}\left(I_{0}^{\vartheta+1-n C} D_{0}^{\vartheta} \varkappa(t)\right) .
\end{aligned}
$$

Then

$$
I_{1}=-b_{n} I_{T}^{n-\kappa} \Theta(0)-\int_{0}^{T} \frac{d}{d t}\left(I_{0}^{\vartheta+1-n C} D_{0}^{\vartheta} \varkappa(t)\right) \frac{d}{d t} I_{T}^{n-\kappa} \Theta(t) d t
$$

Using integration by parts and Lemma 1, we have that

$$
\begin{aligned}
I_{1}= & -b_{n} I_{T}^{n-\kappa} \Theta(0)-\left.I_{0}^{\vartheta+1-n C} D_{0}^{\vartheta} \varkappa(t) \frac{d}{d t} I_{T}^{n-\kappa} \Theta(t)\right|_{t=0} ^{T} \\
& +\int_{0}^{T} I_{0}^{\vartheta+1-n C} D_{0}^{\vartheta} \varkappa(t) \frac{d^{2}}{d t^{2}} I_{T}^{n-\kappa} \Theta(t) d t \\
= & -b_{n} I_{T}^{n-\kappa} \Theta(0)+\int_{0}^{T} I_{0}^{\vartheta+1-n C} D_{0}^{\vartheta} \varkappa(t) \frac{d^{2}}{d t^{2}} I_{T}^{n-\kappa} \Theta(t) d t \\
= & -b_{n} I_{T}^{n-\kappa} \Theta(0)+\int_{0}^{T}{ }^{C} D_{0}^{\vartheta} \varkappa(t) I_{T}^{\vartheta+1-n} \frac{d^{2}}{d t^{2}} I_{T}^{n-\kappa} \Theta(t) d t .
\end{aligned}
$$

Note that

$$
\left.I_{0}^{\vartheta+1-n C} D_{0}^{\vartheta} \varkappa(t) \frac{d}{d t} I_{T}^{n-\kappa} \Theta(t)\right|_{t=T}=0, \quad \varsigma>1+\kappa-n
$$

and

$$
\begin{aligned}
\left.I_{0}^{\vartheta+1-n C} D_{0}^{\vartheta} \varkappa(t) \frac{d}{d t} I_{T}^{n-\kappa} \Theta(t)\right|_{t=0} & =\left.I_{0}^{\vartheta+1-n} I_{0}^{n-\vartheta} \varkappa^{(n)}(t) \frac{d}{d t} I_{T}^{n-\kappa} \Theta(t)\right|_{t=0} \\
& =\left.I_{0}^{1} \varkappa^{(n)}(t) \frac{d}{d t} I_{T}^{n-\kappa} \Theta(t)\right|_{t=0} \\
& =\left.\left(\varkappa^{(n-1)}(t)-\varkappa^{(n-1)}(0)\right) \frac{d}{d t} I_{T}^{n-\kappa} \Theta(t)\right|_{t=0}=0 .
\end{aligned}
$$

Next, we insert $t^{\eta / m} \Theta(t)^{1 / m} t^{-\eta / m} \Theta(t)^{-1 / m}$ inside the integral of (15):

$$
I_{1}=-b_{n} I_{T}^{n-\kappa} \Theta(0)+\int_{0}^{T}{ }^{C} D_{0}^{\vartheta} \varkappa(t) \Theta(t)^{1 / m} t^{\eta / m} \Theta(t)^{-1 / m}(t) t^{-\eta / m} I_{T}^{\vartheta+1-n} \frac{d^{2}}{d t^{2}} I_{T}^{n-\kappa} \Theta(t) d t .
$$

Using the $\varepsilon$-Young inequality with $0<\varepsilon<1 / 2$, we obtain

$$
\begin{aligned}
I_{1} \leq & -b_{n} I_{T}^{n-\kappa} \Theta(0)+\varepsilon \int_{0}^{T}\left|{ }^{C} D_{0}^{\vartheta} \varkappa(t)\right|^{m} \Theta(t) t^{\eta} d t \\
& +K(\varepsilon, m) \int_{0}^{T} \Theta(t)^{-m^{\prime} / m} t^{-\eta m^{\prime} / m}\left(I_{T}^{\vartheta+1-n} \frac{d^{2}}{d t^{2}} I_{T}^{n-\kappa} \Theta(t)\right)^{m^{\prime}} d t, \quad K(\varepsilon, m)>0 .
\end{aligned}
$$


By Lemmas 5 and 6 we have

$$
\begin{aligned}
I_{1} \leq & -b_{n} \frac{\Gamma(\varsigma+1)}{\Gamma(n-\kappa+\varsigma+1)} T^{n-\kappa}+\varepsilon \int_{0}^{T}\left|{ }^{C} D_{0}^{\vartheta} \varkappa(t)\right|^{m} \Theta(t) t^{\eta} d t \\
& +K(\varepsilon, m) K_{\vartheta+1-n, n-\kappa}^{\eta, m^{\prime}, \varsigma} T^{\eta\left(1-m^{\prime}\right)+m^{\prime}(\vartheta-\kappa-1)+1} .
\end{aligned}
$$

Similarly,

$$
\begin{aligned}
I_{2} \leq & -b_{n} \frac{\Gamma(\varsigma+1)}{\Gamma(n-v+\varsigma+1)} T^{n-v}+\varepsilon \int_{0}^{T}\left|{ }^{C} D_{0}^{\vartheta} \varkappa(t)\right|^{m} \Theta(t) t^{\eta} d t \\
& +K(\varepsilon, m) K_{\vartheta+1-n, n-v}^{\eta, m^{\prime}, \varsigma} T^{\eta\left(1-m^{\prime}\right)+m^{\prime}(\vartheta-v-1)+1} .
\end{aligned}
$$

Hence from (12), (16), and (17) it follows that

$$
\begin{aligned}
(1- & 2 \varepsilon) I+\Gamma(\varsigma+1) b_{n} T^{n-v}\left(\frac{T^{v-\kappa}}{\Gamma(n-\kappa+\varsigma+1)}+\frac{1}{\Gamma(n-v+\varsigma+1)}\right) \\
\leq & K(\varepsilon, m) K_{\vartheta+1-n, n-\kappa}^{\eta, m^{\prime}, \varsigma} T^{\eta\left(1-m^{\prime}\right)+m^{\prime}(\vartheta-\kappa-1)+1} \\
& +K(\varepsilon, m) K_{\vartheta+1-n, n-v}^{\eta, m^{\prime},} T^{\eta\left(1-m^{\prime}\right)+m^{\prime}(\vartheta-v-1)+1} .
\end{aligned}
$$

Since $b_{n}>0$ and $0<\varepsilon<1 / 2$, we find

$$
\Gamma(\varsigma+1) b_{n} T^{n-v}\left(\frac{T^{v-\kappa}}{\Gamma(n-\kappa+\varsigma+1)}+\frac{1}{\Gamma(n-v+\varsigma+1)}\right) \geq \frac{\Gamma(\varsigma+1)}{\Gamma(n-v+\varsigma+1)} b_{n} T^{n-v}
$$

and

$$
\begin{aligned}
(1-2 \varepsilon) & \int_{0}^{T} \Theta(t) t^{\eta}\left|{ }^{C} D_{0}^{\vartheta} \varkappa(t)\right|^{m} d t \\
& +\Gamma(\varsigma+1) b_{n} T^{n-v}\left(\frac{T^{v-\kappa}}{\Gamma(n-\kappa+\varsigma+1)}+\frac{1}{\Gamma(n-v+\varsigma+1)}\right) \\
\geq & \Gamma(\varsigma+1) b_{n} T^{n-v}\left(\frac{T^{v-\kappa}}{\Gamma(n-\kappa+\varsigma+1)}+\frac{1}{\Gamma(n-v+\varsigma+1)}\right) \\
\geq & \frac{\Gamma(\varsigma+1)}{\Gamma(n-v+\varsigma+1)} b_{n} T^{n-v} .
\end{aligned}
$$

Therefore

$$
C_{1} b_{n} T^{n-v} \leq C_{2} T^{\eta\left(1-m^{\prime}\right)+m^{\prime}(\vartheta-\kappa-1)+1}+C_{3} T^{\eta\left(1-m^{\prime}\right)+m^{\prime}(\vartheta-v-1)+1},
$$

where

$$
C_{1}=\frac{\Gamma(\varsigma+1)}{\Gamma(n-v+\varsigma+1)}, \quad C_{2}=K(\varepsilon, m) K_{\vartheta+1-n, n-\kappa}^{\eta, m^{\prime}, \varsigma}, \quad C_{3}=K(\varepsilon, m) K_{\vartheta+1-n, n-v}^{\eta, m^{\prime}, \varsigma},
$$

or

$$
b_{n} \leq \frac{1}{C_{1}} T^{v-n}\left(C_{2} T^{\eta\left(1-m^{\prime}\right)+m^{\prime}(\vartheta-\kappa-1)+1}+C_{3} T^{\eta\left(1-m^{\prime}\right)+m^{\prime}(\vartheta-v-1)+1}\right)
$$




$$
=\frac{1}{C_{1}} T^{v-n+\eta\left(1-m^{\prime}\right)+m^{\prime}(\vartheta-v-1)+1}\left(C_{2} T^{m^{\prime}(v-\kappa)}+C_{3}\right) .
$$

Note that $v-n+\eta\left(1-m^{\prime}\right)+m^{\prime}(\vartheta-v-1)+1<0$ and $m^{\prime}(v-\kappa)<0$, and, consequently, $T^{v-n+\eta\left(1-m^{\prime}\right)+m^{\prime}(\vartheta-v-1)+1}, T^{m^{\prime}(v-\kappa)} \rightarrow 0$ as $T \rightarrow \infty$. Therefore

$$
b_{n} \leq 0
$$

We get a contradiction since $b_{n}>0$.

Theorem 2 Let $m(\vartheta-v)-1<\eta<m-1, m>1$, and $b_{n}=0$. Then the only global solution to problem (11) is

$$
\varkappa(t)=c_{0}+c_{1} t+\cdots+\frac{c_{n-1}}{(n-1) !} t^{n-1}, \quad t>0 .
$$

Proof Taking $b_{n}=0$ in (18), we find

$$
(1-2 \varepsilon) I \leq C_{2} T^{\eta\left(1-m^{\prime}\right)+m^{\prime}(\vartheta-\kappa-1)+1}+C_{3} T^{\eta\left(1-m^{\prime}\right)+m^{\prime}(\vartheta-v-1)+1},
$$

or

$$
I \leq C_{4} T^{\eta\left(1-m^{\prime}\right)+m^{\prime}(\vartheta-v-1)+1}\left(C_{2} T^{m^{\prime}(v-\kappa)}+C_{3}\right)
$$

where $C_{4}=\frac{1}{1-2 \varepsilon}$ and $0<\varepsilon<1 / 2$. Using (10) and (12), we get

$$
\begin{aligned}
\int_{0}^{T}\left(1-\frac{t}{T}\right)^{\zeta} t^{\eta}\left|{ }^{C} D_{0}^{\vartheta} \varkappa(t)\right|^{m} d t \leq & C_{4} T^{\eta\left(1-m^{\prime}\right)+m^{\prime}(\vartheta-v-1)+1} \\
& \times\left(C_{2} T^{m^{\prime}(v-\kappa)}+C_{3}\right) .
\end{aligned}
$$

Since $v \leq \kappa$ and $m(\vartheta-v)-1<\eta$, we have $m^{\prime}(v-\kappa) \leq 0$ and $\eta\left(1-m^{\prime}\right)+m^{\prime}(\vartheta-v-1)+1<0$. Taking the limit as $T \rightarrow \infty$ in (20) and using Fatou's lemma, we obtain

$$
\int_{0}^{T} t^{\eta}\left|{ }^{C} D_{0}^{\vartheta} \varkappa(t)\right|^{m} d t=0
$$

which yields

$$
{ }^{C} D_{0}^{\vartheta} \varkappa(t)=0, \quad t>0
$$

Then by Lemma 4 we have

$$
\varkappa(t)=c_{0}+c_{1} t+\cdots+\frac{c_{n-1}}{(n-1) !} t^{n-1}, \quad t>0 .
$$

\section{Examples}

In this section, we give some examples to justify the preceding results. 
Example 1 The fractional differential problem

$$
\begin{aligned}
& { }^{C} D_{0}^{1.8} \varkappa(t)+{ }^{C} D_{0}^{1.5} \varkappa(t) \geq t^{0.5}\left|{ }^{C} D_{0}^{0.6} \varkappa(t)\right|^{2}, \quad t>0, \\
& \varkappa(0)=0, \quad \varkappa^{\prime}(0)=1,
\end{aligned}
$$

is a particular case of (11) where $\kappa=0.8, v=0.5, \eta=0.5, \vartheta=0.6, m=2, b_{0}=0$, and $b_{1}=1$. Therefore by Theorem 1 the fractional differential problem (21) has no nontrivial global solutions in $A C^{2}[0, \infty)$.

Example 2 The fractional differential problem

$$
\begin{aligned}
& { }^{C} D_{0}^{2.7} \varkappa(t)+{ }^{C} D_{0}^{2.4} \varkappa(t) \geq t^{-0.4}\left|{ }^{C} D_{0}^{1.5} \varkappa(t)\right|^{3}, \quad t>0, \\
& \varkappa(0)=1, \quad \varkappa^{\prime}(0)=-2, \quad \varkappa^{\prime \prime}(0)=3,
\end{aligned}
$$

is a particular case of (11) where $\kappa=1.7, v=1.4, \eta=-0.4, \vartheta=1.5, m=3$ and $b_{0}=1$, $b_{1}=-2, b_{2}=3$. Therefore, by Theorem 1 , the fractional differential problem (22) has no nontrivial global solution in $A C^{3}[0, \infty)$.

Example 3 The fractional differential problem

$$
\begin{aligned}
& { }^{C} D_{0}^{3.7} \varkappa(t)+{ }^{C} D_{0}^{3.5} \varkappa(t) \geq t^{0.8}\left|{ }^{C} D_{0}^{2.6} \varkappa(t)\right|^{3}, \quad t>0, \\
& \varkappa(0)=4, \quad \varkappa^{\prime}(0)=1, \quad \varkappa^{\prime \prime}(0)=2, \quad \varkappa^{\prime \prime \prime}(0)=0,
\end{aligned}
$$

is a special case of (11) when $\kappa=2.7, v=2.5, \eta=0.8, \vartheta=2.6, m=3, b_{0}=4, b_{1}=1, b_{2}=2$, and $b_{3}=0$. Therefore by Theorem 2 the only global solution to problem (23) is

$$
\varkappa(t)=4+t+t^{2}, \quad t>0 .
$$

\section{Concluding remarks}

In this paper, we studied a new class of fractional differential inequalities involving the Caputo fractional derivatives depending on two different orders. With the aid of the test function technique and some properties of fractional integrals, we investigated the nonexistence of nontrivial global solutions in a suitable space. Three simulation examples were presented to illustrate our acquired results. Moreover, for the telegraphing problem [38], it was deduced that the solutions approach the solution of the same problem without the $n$th derivative as $t \rightarrow \infty$. This result was summed up and generalized to the FD. We investigated the case in which there is a lower-order FD in the inequality (or equation), for example, problem (2). We realized how effective $D_{0}^{v} \varkappa$ would be on the blow-up phenomenon. Specifically, it affected the range of values $m$ which guaranteed blow-ups in finite time Based on this, we arrived at the conclusion that the lower-order derivative (i.e., $v$ ) determines the range of blow-up much the same as the parabolic portion and hyperbolic problem. The presented results are more general than those in the literature, which can be obtained as particular cases: for more detail, see Remarks 2, 3, and 4.

In future work, many cases can be established for more general operators containing another function, for instance, the generalized Caputo [39] or Hilfer [40] fractional operator. Also, it will be of interest to study the problem of this paper for the Mittag-Leffler power low [41]. 


\section{Acknowledgements}

The first and second authors are grateful for the support provided by Imam Abdulrahman Bin Faisal University.

\section{Funding}

Not applicable.

\section{Abbreviations}

Not applicable.

Availability of data and materials

Not applicable.

\section{Competing interests}

The authors declare that they have no competing interests.

\section{Authors' contributions}

All authors make equal contributions, read, and supported the last original copy.

\section{Author details}

'Department of Basic Engineering Sciences, College of Engineering, Imam Abdulrahman Bin Faisal University, P.O. Box 1982, Dammam, 34151, Saudi Arabia. ${ }^{2}$ Department of Mathematics, Hodeidah University, AL-Hodeidah, Yemen. ${ }^{3}$ Department of Mathematics, Cankaya University, 06790 Etimesgut, Ankara, Turkey. ${ }^{4}$ Medical University Hospital, China Medical University, Taichung, Taiwan.

\section{Publisher's Note}

Springer Nature remains neutral with regard to jurisdictional claims in published maps and institutional affiliations.

Received: 17 February 2021 Accepted: 27 April 2021 Published online: 08 May 2021

\section{References}

1. Agarwal, R.P., Benchohra, M., Hamani, S.A.: Survey on existence results for boundary value problems of nonlinear fractional differential equations and inclusions. Acta Appl. Math. 109(3), 973-1033 (2010)

2. Furati, K.M., Kassim, M.D., Tatar, N.-E.: Existence and uniqueness for a problem involving Hilfer fractional derivative. Comput. Math. Appl. 64, 1616-1626 (2012)

3. Jarad, F., Abdeljawad, T., Hammouch, Z:: On a class of ordinary differential equations in the frame of Atangana-Baleanu fractional derivative. Chaos Solitons Fractals 117, 16-20 (2018)

4. Kilbas, A.A., Srivastava, H.M., Trujillo, J.J.: Theory and Applications of Fractional Differential Equations, vol. 204. Elsevier Science, Amsterdam (2006)

5. Podlubny, I.: Fractional Differential Equations. Academic Press, San Diego (1999)

6. Samko, S.G., Kilbas, A.A., Marichev, O.I.: Fractional Integrals and Derivatives: Theory and Applications. Gordon and Breach, Switzerland (1993)

7. Maraaba, T.A., Jarad, F., Baleanu, D.: On the existence and the uniqueness theorem for fractional differential equations with bounded delay within Caputo derivatives. Sci. China Ser. A 51(10), 1775-1786 (2008)

8. Abdo, M.S., Shah, K., Panchal, S.K., Wahash, H.A.: Existence and Ulam stability results of a coupled system for terminal value problems involving $\psi$-Hilfer fractional operator. Adv. Differ. Equ. 2020(1), 1 (2020)

9. Abdo, M.S., Abdeljawad, T., Ali, S.M., Shah, K., Jarad, F.: Existence of positive solutions for weighted fractional order differential equations. Chaos Solitons Fractals 141, 110341 (2020)

10. Abdo, M.S., Abdeljawad, T., Shah, K., Jarad, F.: Study of impulsive problems under Mittag-Leffler power law. Heliyon 6(10), e05109 (2020)

11. Abdo, M.S.: Further results on the existence of solutions for generalized fractional quadratic functional integral equations. J. Math. Anal. Model. 1(1), 33-46 (2020)

12. Abdo, M.S., Thabet, S.T., Ahmad, B.: The existence and Ulam-Hyers stability results for $\psi$-Hilfer fractional integrodifferential equations. J. Pseudo-Differ. Oper. Appl. 11(4), 1757-1780 (2020)

13. Kassim, M.D., Tatar, N.-E.: Well-posedness and stability for a differential problem with Hilfer-Hadamard fractional derivative. Abstr. Appl. Anal. 2013, 1 (2013)

14. Messaoudi, S.A., Said-Houari, B., Tatar, N.-E.: Global existence and asymptotic behavior for a fractional differential equation. Appl. Math. Comput. 188, 1955-1962 (2007)

15. Tatar, N.-E.: Existence results for an evolution problem with fractional nonlocal conditions. Comput. Math. Appl. 60, 2971-2982 (2010)

16. Kassim, M.D., Furati, K.M., Tatar, N.-E.: Asymptotic behavior of solutions to nonlinear fractional differential equations. Math. Model. Anal. 21(5), 610-629 (2016)

17. Kassim, M.D., Furati, K.M., Tatar, N.-E.: Asymptotic behavior of solutions to nonlinear initial-value fractional differential problems. Electron. J. Differ. Equ. 2016, 1 (2016)

18. Kassim, M.D., Tatar, N.-E.: Convergence of solutions of fractional differential equations to power-type functions. Electron. J. Differ. Equ. 2020, 1 (2020)

19. Kassim, M.D., Tatar, N.-E.: Stability of logarithmic type for a Hadamard fractional differential problem. J. Pseudo-Differ. Oper. Appl. 2020, 447-466 (2020)

20. Alqahtani, B., Aydi, H., Karapınar, E., Rakočević, V.: A solution for Volterra fractional integral equations by hybrid contractions. Mathematics 7(8), 1-10 (2019)

21. Baleanu, D., Etemad, S., Rezapour, S.: A hybrid Caputo fractional modeling for thermostat with hybrid boundary value conditions. Bound. Value Probl. 2020(1), 1 (2020) 
22. Baleanu, D., Jajarmi, A., Mohammadi, H., Rezapour, S.A.: New study on the mathematical modelling of human liver with Caputo-Fabrizio fractional derivative. Chaos Solitons Fractals 134, 109705 (2020)

23. Karapınar, E., Fulga, A., Rashid, M., Shahid, L., Aydi, H.: Large contractions on quasi-metric spaces with an application to nonlinear fractional differential equations. Mathematics 7(5), 1-11 (1019)

24. Mohammadi, H., Kumar, S., Rezapour, S., Etemad, S.: A theoretical study of the Caputo-Fabrizio fractional modeling for hearing loss due to Mumps virus with optimal control. Chaos Solitons Fractals 144, 110668 (2021)

25. Rezapour, S., Mohammadi, H., Jajarmi, A.: A new mathematical model for Zika virus transmission. Adv. Differ. Equ. 2020(1), 1 (2020)

26. Tuan, N.H., Mohammadi, H., Rezapour, S.: A mathematical model for COVID-19 transmission by using the Caputo fractional derivative. Chaos Solitons Fractals 140, 110107 (2020)

27. Furati, K.M., Kassim, M.D., Tatar, N.E.: Non-existence of global solutions for a differential equation involving Hilfer fractional derivative. Electron. J. Differ. Equ. 2013, 235 (2013)

28. Kassim, M.D., Furati, K.M., Tatar, N.-E.: On a differential equation involving Hilfer-Hadamard fractional derivative. Abstr. Appl. Anal. 2012, 1 (2012)

29. Laskri, Y., Tatar, N.E.:. The critical exponent for an ordinary fractional differential problem. Comput. Math. Appl. 59 $1266-1270(2010)$

30. Tatar, N.-E.: Nonexistence results for a fractional problem arising in thermal diffusion in fractal media. Chaos Solitons Fractals 36, 1205-1214 (2008)

31. Kirane, M., Medved, M., Tatar, N.E.: On the nonexistence of blowing-up solutions to a fractional functional differential equations. Georgian Math. J. 19, 127-144 (2012)

32. Kirane, M., Tatar, N.-E.: Nonexistence of solutions to a hyperbolic equation with a time fractional damping. Z. Anal. Anwend. 25, 131-142 (2006)

33. Kirane, M., Tatar, N.-E.: Absence of local and global solutions to an elliptic system with time-fractional dynamical boundary conditions. Sib. Math. J. 48(3), 477-488 (2007)

34. Kirane, M., Laskri, Y., Tatar, N.E.: Critical exponents of Fujita type for certain evolution equations and systems with spatio-temporal fractional derivatives. J. Math. Anal. Appl. 312(2), 488-501 (2005)

35. Kassim, M.D., Furati, K.M., Tatar, N.-E.: Nonexistence of global solutions for a fractional differential problem. J. Comput. Appl. Math. 314, 61-68 (2017)

36. Kassim, M.D., Furati, K.M., Tatar, N.-E.: Non-existence for fractionally damped fractional differential problems. Acta Math. Sci. 37(1), 119-130 (2017)

37. Jleli, M., Samet, B.: Nonexistence results for some classes of nonlinear fractional differential inequalities. J. Funct. Spaces 2020, 1-8 (2020)

38. Cascaval, R.C., Eckstein, E.C., Frota, C.L., Godstein, J.A.. Fractional telegraph equations. J. Math. Anal. Appl. 276(1), 145-159 (2020)

39. Almeida, R.: A Caputo fractional derivative of a function with respect to another function. Commun. Nonlinear Sci. Numer. Simul. 44, 460-481 (2017)

40. Sousa, J.V.C., Oliveira, E.C.: On the $\psi$-Hilfer fractional derivative. Commun. Nonlinear Sci. Numer. Simul. 60, 72-91 (2018)

41. Atangana, A., Baleanu, D.: New fractional derivative with non-local and non-singular kernel. Therm. Sci. 20(2), 757-763 (2016)

\section{Submit your manuscript to a SpringerOpen ${ }^{\circ}$ journal and benefit from:}

- Convenient online submission

- Rigorous peer review

- Open access: articles freely available online

- High visibility within the field

- Retaining the copyright to your article

Submit your next manuscript at $\gg$ springeropen.com 